gebeugten Wellen keine merklichen Phasenbeziehungen miteinander haben. Bei einer solchen diskontinuierlichen Raumerfüllung hat man ein Ansteigen der Streuintensität bei Annäherung an den Streuwinkel $0 \mathrm{zu}$ erwarten ${ }^{8}$; beobachtet wird aber ein starker Abfall (Abb.1), wie er für glasartige und flüssige Stoffe kennzeichnend ist. Eine direkte Widerlegung der Anschauung des ,Tetraederpulvers" ist das Auftreten einer ausgeprägten zweiten Koordinationsgruppe in der erhaltenen Atomverteilung (Abb. 2). Die Ordnungsbezirke in der amorphen Phase müssen also größer sein als ein einzelnes Tetraeder.

Wenn man an dem Gedanken des Tetraeders als Baueinheit der Atomanordnung des amorphen Germanium festhalten will, so hat man die Forderung einer gegenseitigen Verkettung der Tetraeder hinzuzufügen. Als Vorbild kann die Verknüpfung der $\mathrm{SiO}_{2}$-Tetraeder im Glas dienen (Warren und Mitarbb. ${ }^{9}$ ). Die Form der Verkettung muß aber beim Germanium wegen der Gleichheit der beteiligten Atome eine andere sein. Das in Abb. 5 wiedergegebene Modell genügt den beiden Bedingungen einer Nahordnung gemäß den Ergebnissen der Fourier-Analyse und einer regellosen Verteilung für größere Abstände. Die beiden Tetraeder $2,3,4,5$ und $1,6,7,8$ sind so gezeichnet, wie sie im Gitter zueinander orientiert sind. Der Schwerpunkt 1 des einen Tetraeders ist die gemeinsame Ecke der 4 Nachbartetraeder, von denen in Abb. 5 nur eines eingetragen ist. Denkt man sich nun die beiden Tetraeder um die Achse 1-2 beliebig gegeneinander verdreht, so werden

${ }^{8} \mathrm{Vgl}$. z. B. B. E. Warren, J. chem. Physics 2, 551 [1934], u. J. Biscoe u. B. E. Warren, J. appl. Physics 13, 364 [1942]. die Abstände und die Zahl der Nachbarn des Atomes 1 in erster und zweiter Koordination nicht geändert; darüber hinaus tritt aber rasch eine regellose Orientierung und damit eine ungeordnete Atomverteilung auf.

Bei der Umwandlung der amorphen Phase in die kristalline durch Wärmezufuhr erfolgt ein

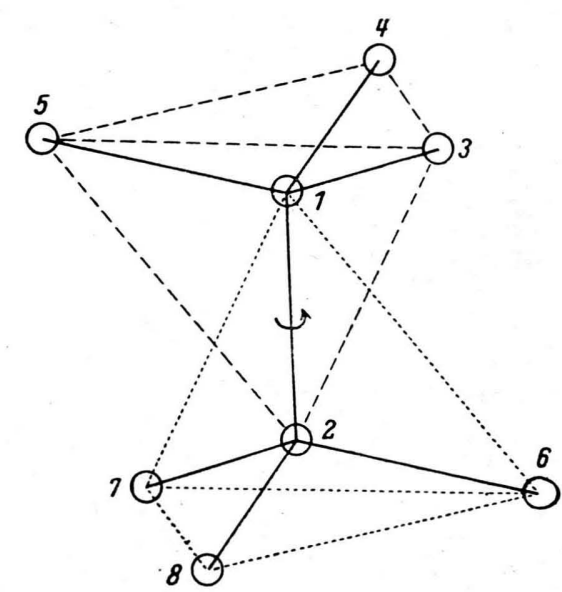

Abb. 5. Orientierung der Tetraeder in amorphem Germanium.

Ordnungsvorgang, bei dem die einzelnen Tetraeder in die durch die Symmetriegesetze des Gitters vorgeschriebenen Lagen sich einstellen. Die im Gitter vorhandene Atomanordnung bleibt als Nahordnung im amorphen Zustand erhalten, geht aber beim Aufschmelzen völlig verloren, wie die beobachtete Änderung der Koordinationszahl und der Abstände zweifelsfrei ergibt.

9 B. E. Warren, H. Krutter u. O. Morningst a r, J. Amer. Soc. 19, 202 [1936], u. B. E. W a r r en, J. Amer. ceram. Soc. 24, 256 [1941].

\title{
Zur Rückdiffusion schneller Elektronen
}

\section{Von Walther Bothe}

Aus dem Physikalischen Institut der Universität Heidelberg und dem Institut für Physik im Kaiser-Wilhelm-Institut für medizinische Forschung, Heidelberg

(Z. Naturforschg. 4 a, 542-545 [1949]; eingegangen am 20. März 1949)

Für Elektronen von 370 und $680 \mathrm{keV}$ wurde die Rückdiffusion an $\mathrm{C}, \mathrm{Al}, \mathrm{Cu}, \mathrm{Sn}$ und $\mathrm{Pb}$ untersucht. Die relativen Intensitäten und die Energiespektren der rückdiffundierten Elektronen wurden ausgemessen. Die Ergebnisse sind in den Abb. 2 bis 4 dargestellt und kurz diskutiert.

1. Ziel und Anordnung der Versuche Ü ber die Rückdiffusion schneller Elektronen aus dicken Wänden liegt eine Reihe älterer Untersuchungen vor ${ }^{1}$. Die wichtigen Punkte sind der „Rückdiffusionskoeffizient“, d.h. der Bruchteil der auffallenden Elektronen, der wieder aus der Wand austritt, und die Energieverteilung der

1 Vgl. Hb. Physik XII, 2, S. 25 [1933]. 
rückdiffundierten Elektronen. Letztere ist mit homogenen Primärstrahlen bisher nur in dem Energiebereich von $40 \mathrm{keV}$ abwärts und meist nur qualitativ untersucht worden. Wenn auch grundsätzlich Neues von der näheren Untersuchung der Rückdiffusion kaum zu erwarten ist, so sind diese Fragen doch mindestens in mancherlei praktischer Hinsicht nicht unwichtig (Konstruktion von Ionisationskammern, Stielstrahlung bei Röntgenröhren, Dosisverteilung bei der Strahlentherapie u. ä.).

Kürzlich haben wir zu Versuchen über Einzelstreuung von Elektronen in dünnen Folien ${ }^{2}$ eine Apparatur gebaut, die auch geeignet ist, die von dicken Schichten rückdiffundierten Elektronen im Energiebereich einiger $100 \mathrm{keV}$ auf ihre Energieverteilung zu untersuchen. Von dieser Möglichkeit wurde Gebrauch gemacht.

Die wesentlichen Bestandteile der Anordnung sind folgende. Als Strahlenquelle dient ein starkes RaD +EPräparat. Ein magnetischer Monochromator sondert aus der kontinuierlichen $\beta$-Strahlung des RaE ein kanalisiertes Bündel von leidlich definierter Energie aus. Dieses trifft unter einem Einfallswinkel von 20 bis $35^{\circ}$ auf eine praktisch unendlich dicke Platte des $\mathrm{zu}$ untersuchenden Stoffes, die am Rande eines magnetischen Halbkreisspektrometers angebracht ist. Die Elektronen, die unter einem Winkel von $\mathrm{rd} .50 \pm 15^{\circ}$ gegen die Normale zurückdiffundieren, werden durch das Spektrometer zerlegt und mit einem Zählrohr gezählt. Das totale Auflösungsvermögen der Apparatur ist so, daß Elektronen, wie sie der Monochromator liefert, falls sie ohne Energieverlust von der Schicht zurückgestreut werden, eine Linie von der relativen Halbwertbreite $9 \%$ ergeben. Bei zu großer Intensität werden, nach Bedecken der Quelle mit Silberfiltern geeigneter Dicke, Anschlußmessungen gemacht.

\section{Ergebnis se}

Es wurden die Energieverteilungen der rück. diffundierten Elektronen an Graphit, Aluminium, Kupfer, Zinn und Blei bei den Primärenergien $E_{0}=370$ und $680 \mathrm{keV}$ aufgenommen. Abb. 1 zeigt Beispiele der unmittelbaren Meßergebnisse für die Primärenergie $370 \mathrm{keV}$. Die eingetragenen Meßpunkte zeigen einen ähnlichen Verlauf, wie er bei wesentlich langsameren Elektronen schon qualitativ bekannt war: von der Primärenergie abwärts einen Anstieg zu einem Maximum und monotonen Abfall bei kleineren Energien. Bei den schwereren Elementen erwies sich das Maximum als so schmal, daß das Auflösungsvermögen der

2 W. B othe, Z. Naturforschg. 4 a, 88 [1949].
Apparatur nicht mehr ausreichte, um unmittelbar seine wirkliche Form zu liefern. Hierfür konnte jedoch korrigiert werden, da die Auflösekurve bekannt war aus den Messungen über Einzelstreuung an dünnen Folien, wo die gestreuten Elektronen sicher genau die Primärenergie hatten ${ }^{2}$. Diese Auflösekurve umfaßt sowohl die Inhomogenität des Primärbündels als auch die Verschmierung durch das endliche Auflösungsvermögen des Spektrometers. Die wahre Verteilungskurve der Energien wurde durch Probieren gefunden, indem aus der vermuteten Kurve und der normierten Auflösekurve die Faltungs-

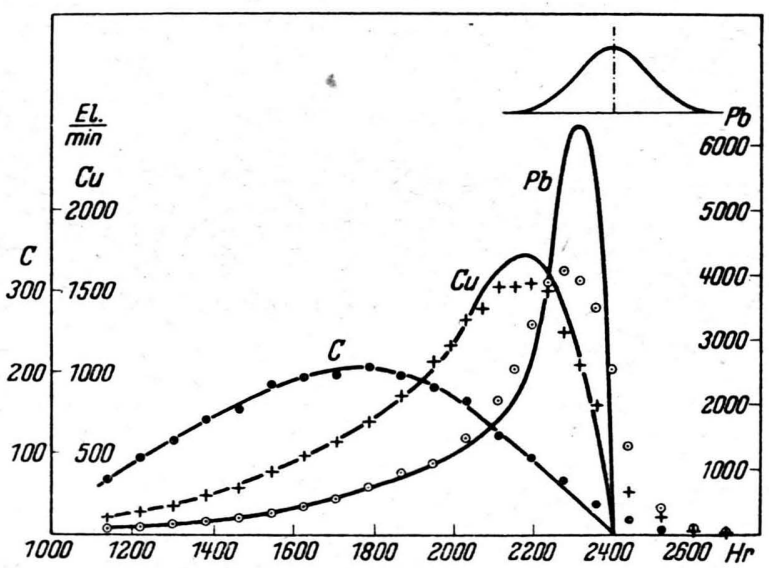

Abb. 1. Meßbeispiele. Die Kurven sind mit der oben eingezeichneten Auflösekurve korrigiert.

integrale für einzelne Kurvenpunkte graphisch ermittelt wurden, die dann die gemessene Kurve ergeben mußten. Das Verfahren ist etwas mühsam, dürfte aber hinreichend genau sein. Die so korrigierten Kurven sind in Abb. 1 eingezeichnet.

Die auf diese Weise erhaltenen Intensitäten stellen erst die Verteilung $N_{\log (H r)} d \log (H r)$ dar, weil die Auflösebreite proportional $\mathrm{Hr}$ ist. Um die Verteilung $N_{H r} d(H r)$ zu erhalten, hätte man noch die Intensitäten durch $\mathrm{Hr}$ zu dividieren. Um die Energieverteilung $N_{E} d E$ zu gewinnen, muß man die gemessenen Intensitäten multiplizieren mit $1 / H r \cdot d(H r) / d E$. Die so erhaltenen Kurven sind in Abb. 2 und 3 wiedergegeben.

Um den Vergleich zu erleichtern, sind als Abszissen Bruchteile der Primärenergie $E_{0}$ aufgetragen. Der Ordinatenmaßstab ist so gewählt, daß das Maximum beim Blei die Höhe 1 hat. Die Kurven wurden nur bis zu $100 \mathrm{keV}$ herab verfolgt, weil bei kleineren Energien die Korrektion für die Ab- 
sorption im Zählerfenster zu unsicher geworden wäre.

Durch Ausplanimetrieren der $N_{H}$, -Kurven wurden die Gesamtzahlen der innerhalb des Öffnungswinkels des Spektrometers ausgesandten Elektronen gefunden. Diese kann man als ,,relative ge-

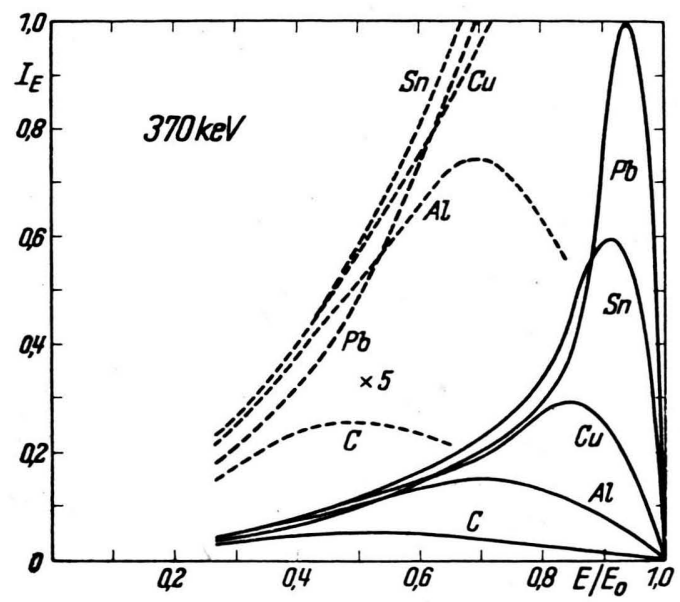

Abb. 2. Energieverteilung der rückdiffundierten Elektronen bei $370 \mathrm{keV}$ Primärenergie.

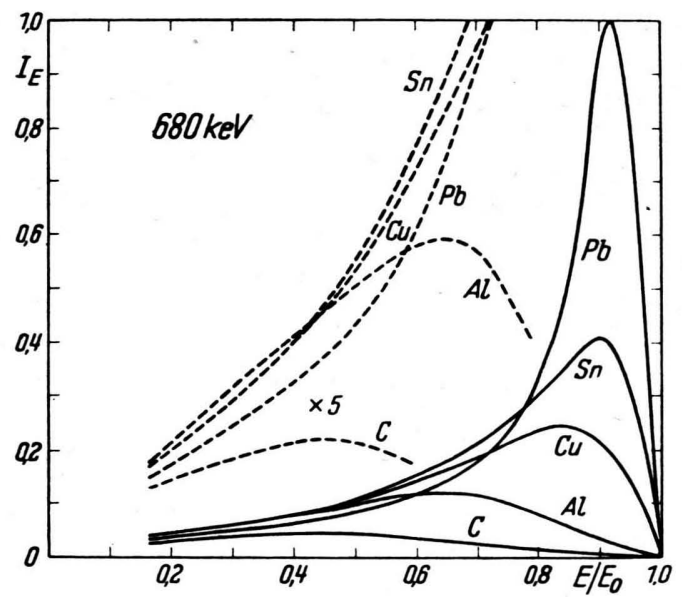

Abb. 3. Energieverteilung der rückdiffundierten Elektronen bei $680 \mathrm{keV}$ Primärenergie.

richtete Rückdiffusionskoeffizienten" auffassen. Sie sind in Abb. 4 aufgetragen, und zwar in solchen Maßstäben, daß die Meßpunkte für die beiden Energien möglichst nahe beieinander liegen.

\section{Diskussion}

Die gerichteten Rückdiffusionskoeffizienten für die beiden Energien (Abb. 4) stimmen in ihrer Abhängigkeit von der Ordnungszahl innerhalb der Meßgenauigkeit überein. Der Verlauf ist auch im wesentlichen derselbe, wie er früher für den über alle Austrittsrichtungen gemessenen ,,diffusen" Rückdiffusionskoeffizienten gefunden worden ist. Hieraus ist zu schließen, daß die Richtungsverteilung der rückdiffundierten Elektronen nicht sehr vom Material abhängt. In Abb. 4 sind zum Vergleich noch die Ergebnisse eingetragen, die $\mathrm{Kovarik}^{3}$ mit den unzerlegten $\beta$-Strahlen des RaE, ohne Zerlegung der rückdiffundierten Elektronen, bei diffusem Einfall und diffusem Austritt, mit einer halbkugelförmigen Ionisationskammer erhalten hat. Die zugehörigen absoluten Rückdiffusionskoeffizienten sind angeschrieben.

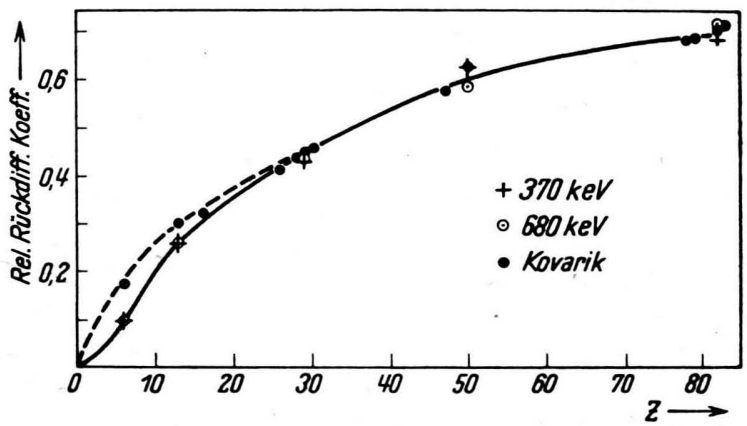

Abb. 4. - Relative „gerichtete“ Rückdiffusionskoeffizienten für 370 und $680 \mathrm{keV}$ Primärenergie. --_- Absolute „diffuse“ Rückdiffusionskoeffizienten für inhomogene RaE-Elektronen nach Kovarik.

Deutliche Abweichungen gegenüber dem von uns gemessenen Verlauf scheinen nur im Gebiet der leichtesten Elemente aufzutreten; unser Meßpunkt für Kohlenstoff liegt wesentlich tiefer. Man könnte zunächst vermuten, daß dies an der verschiedenen Einfalls- und Austrittsgeometrie liegt, oder auch an der Verschiedenheit der Meßmethoden (die Ionisationskammer bevorzugt die energieärmeren Anteile, die beim Kohlenstoff besonders stark vertreten sind; s.w. u.). Aber beides reicht zur Erklärung des Unterschiedes nicht aus, denn Kova$\mathrm{rik}$ und $\mathrm{McKeeha{ } ^ { 4 }}$ haben mit einer der unsrigen ganz ähnlichen Geometrie und ebenfalls mit dem Zähler, nur ohne Zerlegung der Primärstrahlen, denselben Verlauf mit der Ordnungszahl gefunden wie vorher Kovarik. Der Hauptgrund für den Unterschied dürfte darin liegen, daß Kovarik mit inhomogenen Primärstrahlen gearbeitet hat, die einen erheblichen Anteil an

3 A. F. K o varik, Philos. Mag. J. Sci. 20, 849 [1910].

4 A. F. K o varik u. L. W. Mckeehan, Physik. Z. 15, 434 [1914]. 
verhältnismäßig energiearmen Elektronen enthielten. Offenbar verschwindet die Durchbiegung, welche unsere Kurven bei den leichtesten Elementen zeigen, wenn man zu kleinen Primärenergien übergeht. Hierauf deuten auch einige Messungen mit 50-100-keV-Elektronen hin, die gelegentlich in unserem Institut ausgeführt wurden.

Der Vergleich der Energieverteilungskurven von $A b b .2$ und 3 zeigt, daß die relative Lage der Maxima nur wenig von der Primärenergie abhängt. Mit abnehmender Primärenergie rücken die Maxima langsam näher an die Primärenergie heran. Dies paßt gut zu Messungen, die P. B. Wagner ${ }^{5}$ im Bereich von 10 bis $40 \mathrm{keV}$ nach einer photographischen Methode ausgeführt hat; die Verschiebung setzt sich hier z. B. für Aluminium in der Weise fort, daß das Maximum für $40 \mathrm{keV}$ bei $0,85 E_{0}$, für $16 \mathrm{keV}$ bei etwa $0,9 E_{0}$ liegt.

Am auffälligsten ist aber die verschiedene Form der Energieverteilungskurven bei verschiedenen Elementen, Die Intensität der stark gebremsten Elektronen $\left(E<\right.$ etwa $\left.0,3 E_{0}\right)$ ist bei allen Elementen nicht sehr verschieden. Die Lage des Maximums aber versehiebt sich mit zunehmender Ordnungszahl stark nach der Seite größerer Energien. Die Intensität der wenig gebremsten Elektronen $\left(E>\right.$ etwa $0,9 E_{0}$ ) nimmt daher sehr stark mit der Ordnungszahl zu. Dieser Punkt dürfte für manche praktischen Fragen wichtig sein.

$\mathrm{Zu}$ einem qualitativen Verständnis der Hauptzüge dieser Erscheinungen kommt man durch folgende einfache Uberlegung. Der Vorgang besteht in der Hauptsache in einer Vielfachstreuung

5 P. B. Wagner, Physic. Rev. 35, 98 [1930]. mit gleichzeitiger Bremsung. Die Streuung ist im wesentlichen eine Wirkung der Atomkerne und daher proportional $Z^{2}$, während die Bremsung durch die Elektronenhülle verursacht wird und daher nur mit $Z$ geht. Der Wettstreit zwischen diesen beiden Vorgängen entscheidet darüber, wie viele Elektronen wieder zum Austritt kommen und welchen Energieverlust sie inzwischen erlitten haben. Die Vielfachstreuung muß, allgemein gesprochen, bis zu einem großen mittleren $\mathrm{Ab}$ lenkungswinkel fortgeschritten sein, damit Rückdiffusion eintreten kann. Die Bahnstrecke, die hierzu nötig ist (gerechnet in Atomen $/ \mathrm{cm}^{2}$ ), ist in grober Näherung proportional $E_{0}^{2} / Z^{2}$. Der relative Energieverlust pro Atom $/ \mathrm{cm}^{2}$ ist, ebenfalls in erster Näherung, proportional $Z / E_{0}{ }^{2}$ (Whiddingtonsche Regel). Der mittlere relative Energieverlust der wieder austretenden Elektronen wird etwa durch das Produkt dieser beiden Ausdrücke, also $1 / Z$, bestimmt. Der mittlere Energieverlust sollte daher mit steigender Ordnungszahl abnehmen und nahezu unabhängig von der Primärenergie sein, wie es das Experiment tatsächlich zeigt. Aus derselben UUberlegung heraus wird es in leichten Elementen öfter als in schweren vorkommen, daß ein Elektron vollständig abgebremst wird, bevor es den zum Wiederaustritt nötigen Streuwinkel erreicht und wieder zur Oberfläche durchdringt. Damit erklärt sich die Zunahme des Rückdiffusionskoeffizienten mit der Ordnungszahl.

Eingehendere Überlegungen hierzu sollen einer besonderen Mitteilung vorbehalten bleiben ${ }^{6}$.

- W. B othe, Ann. Physik, im Druck.

\title{
Analyse durch Kernumwandlung ${ }^{1}$
}

\section{Von Wolfgang Riezler}

Aus dem Physikalischen Institut der Universität Bonn

(Z. Naturforschg. 4 a, 545-549 [1949]; eingegangen am 16. April 1949)

\begin{abstract}
Es werden Untersuchungen mitgeteilt über den Nachweis kleiner Beimengungen in einem Trägermetall durch Bestrahlung der Proben mit Deuteronen. Für den Nachweis von Kohlenstoff in Eisen ist eine Deuteronenenergie von etwa $4 \mathrm{MeV}$ am günstigsten, die Nachweisgrenze hängt wesentlich von den übrigen Verunreinigungen ab. Sehr empfindlich ist der Nachweis von Silicium oder Natrium in Aluminium. Im letzteren Fall liegt die Nachweisgrenze etwa bei $0,001 \%$.
\end{abstract}

$\mathrm{L}$ äßt man Strahlungen, welche geeignet sind, Kernprozesse hervorzurufen, insbesondere Neutronen oder schnelle Deuteronen, auf Materialproben einwirken, so wird in diesen künstliche Radioaktivität erzeugt. Aus den Halbwertszeiten der entstandenen Aktivitäten kann man in vielen Fällen auf die Elemente schließen, die in den be-

${ }_{1}$ Die vorliegende Arbeit war bereits Anfang 1945 für die Reichsberichte für Physik gesetzt, ist jedoch aus zeitbedingten Gründen nicht mehr erschienen. 